\title{
Supplementary figures for: Evaluating uncertainty in estimates of soil moisture memory with a reverse ensemble approach
}

Dave MacLeod $^{1}$, Hannah Cloke ${ }^{2,3}$, Florian Pappenberger ${ }^{4,5}$, and Antje Weisheimer ${ }^{4,6}$

${ }^{1}$ Atmospheric, Oceanic and Planetary Physics, Department of Physics, University of Oxford, Oxford, UK

${ }^{2}$ Department of Geography and Environmental Science, University of Reading, UK

${ }^{3}$ Department of Meteorology, University of Reading, UK

${ }^{4}$ European Centre for Medium-Range Weather Forecasts, Reading, UK

${ }^{5}$ School of Geographical Sciences, Bristol University. Bristol, UK

${ }^{6}$ Department of Physics, National Centre for Atmospheric Science (NCAS), University of Oxford, Oxford, UK

Correspondence to: Dave MacLeod (macleod@atm.ox.ac.uk) 
May start

Level 1

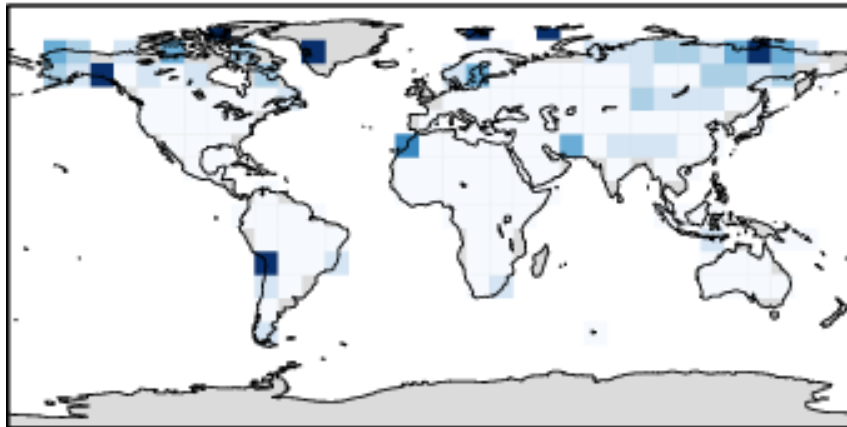

Level 2

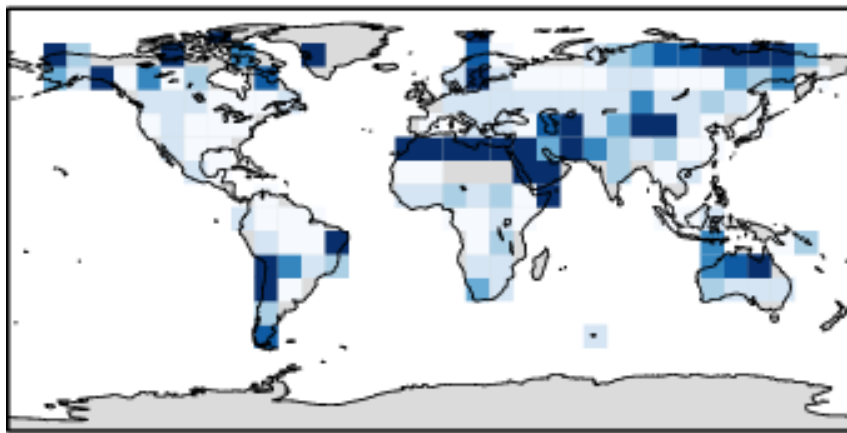

Level 3

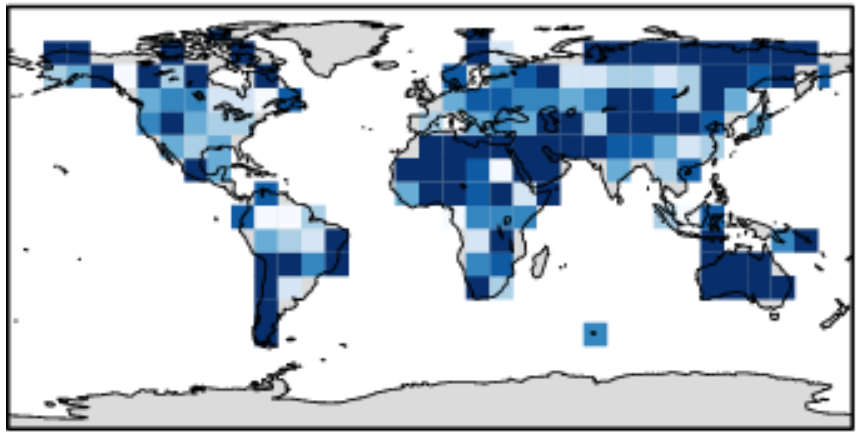

November start

Level 1

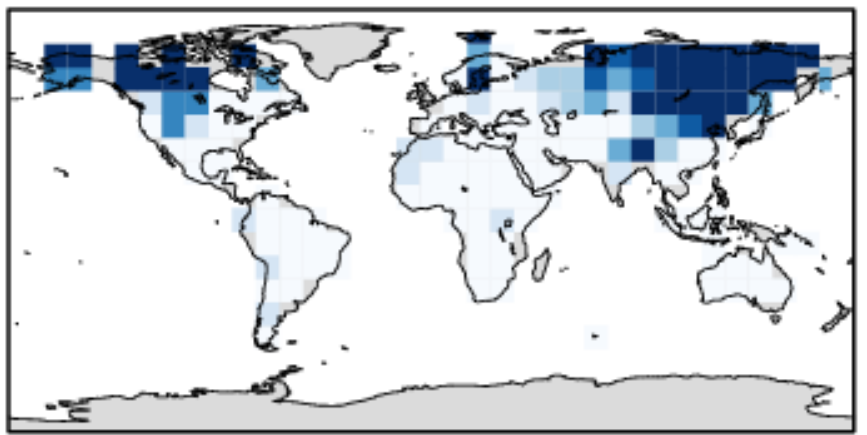

Level 2

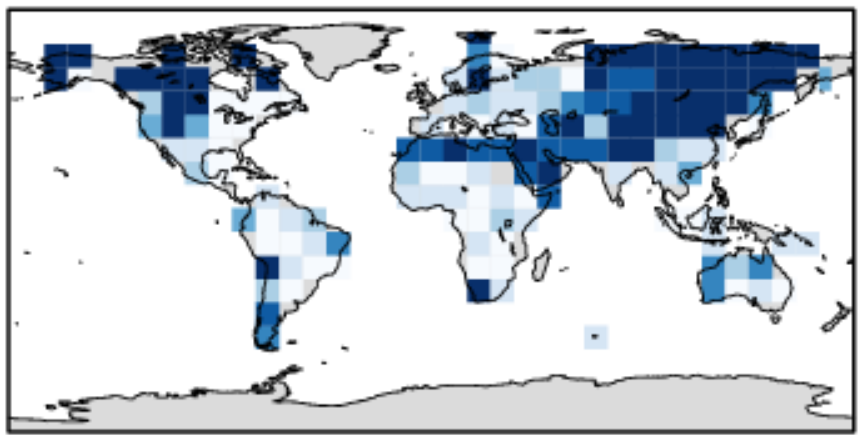

Level 3

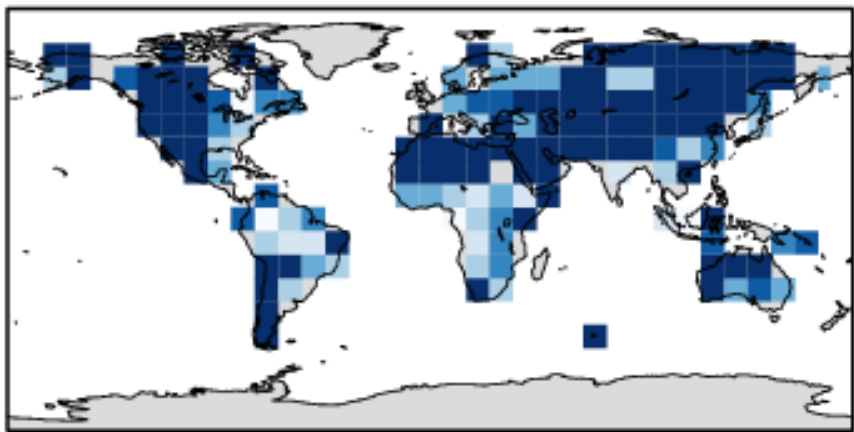

Lead time when $\sigma<\sigma_{0} / \mathrm{e}$ [days]

\begin{tabular}{r|rrrrrr}
\hline & $\mid$ & $\mid$ & $\mid$ & $\mid$ & \\
\hline 20 & 40 & 60 & 80 & 100 & 120
\end{tabular}

Figure 1. Average date of memory loss in the top model soil level, estimated from HTESSEL (default parameter set), for May (left) and November (right) start dates. Grey areas are masked regions of climatologically low soil moisture. 
May start

Level 1

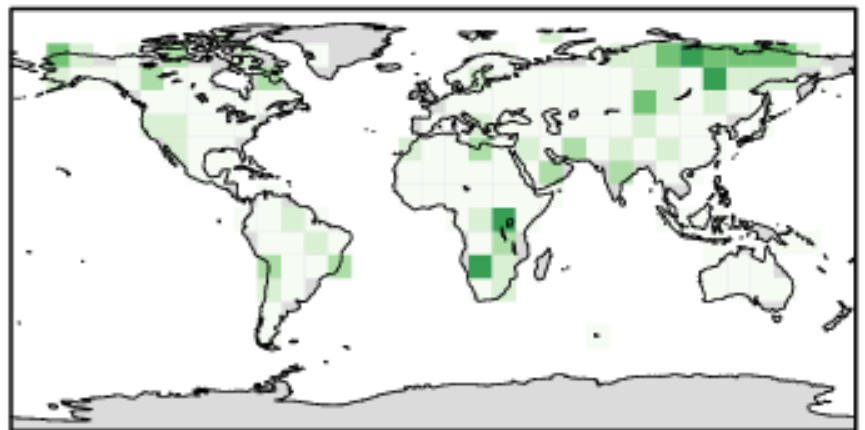

Level 2

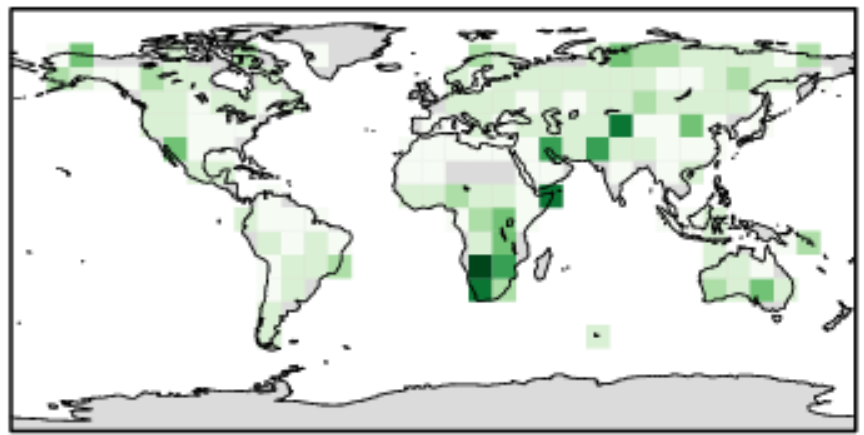

Level 3

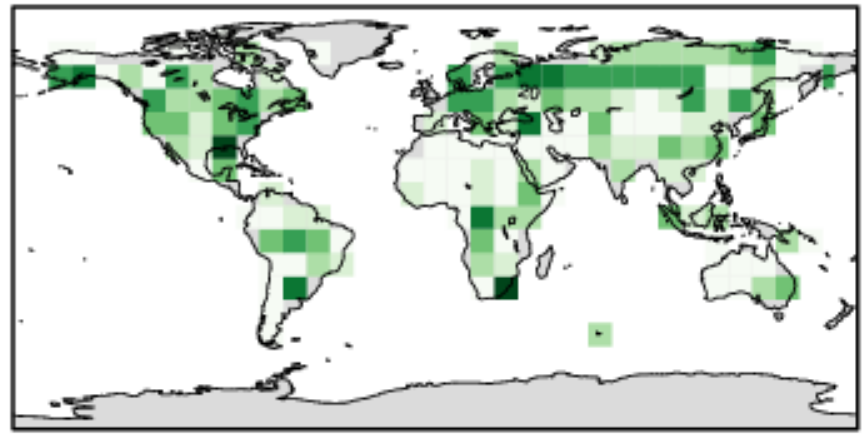

November start

\section{Level 1}

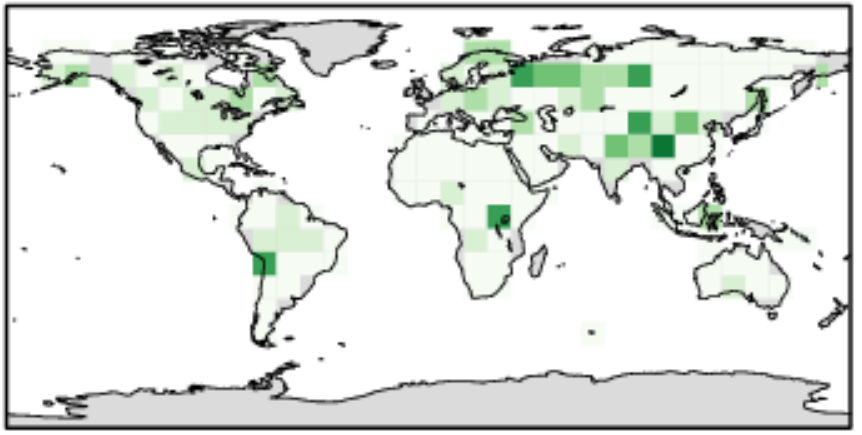

Level 2

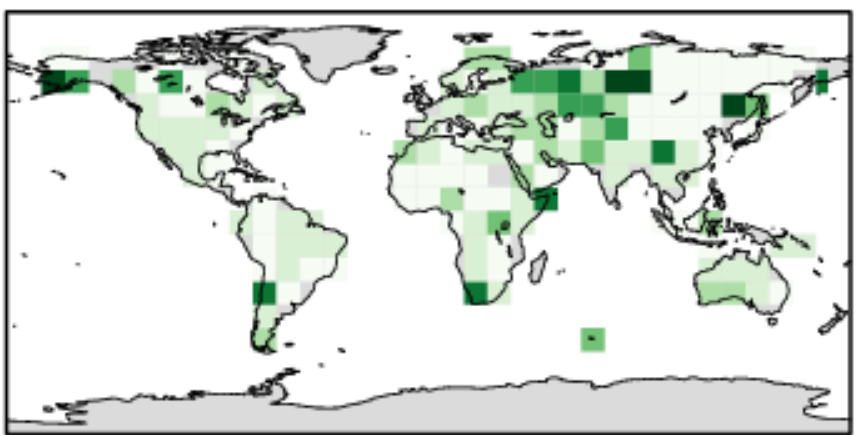

Level 3

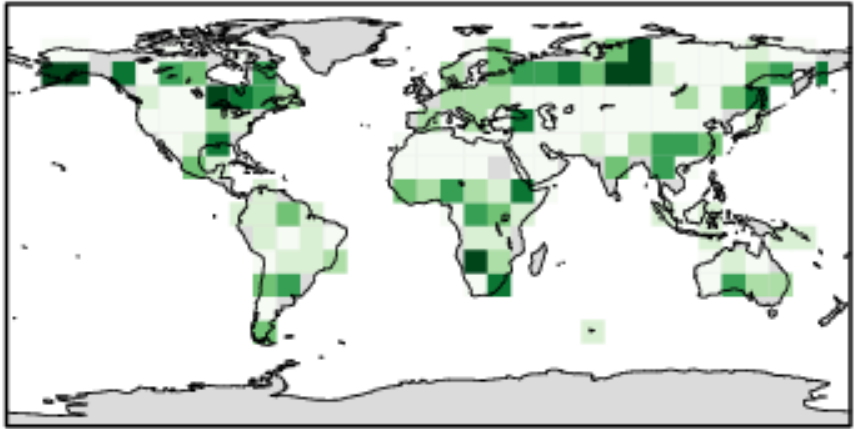

Standard deviation of average memory loss date over parameters [days]

\begin{tabular}{r|rrrrrr}
\hline & $\mid$ & $\mid$ & $\mid$ & $\mid$ & \\
\hline 4 & 12 & 20 & 28 & 36 & 44
\end{tabular}

Figure 2. Standard deviation in the date of memory loss across hydraulic parameters, estimated from HTESSEL, for May (left) and November (right) start dates. 
May start

Level 1

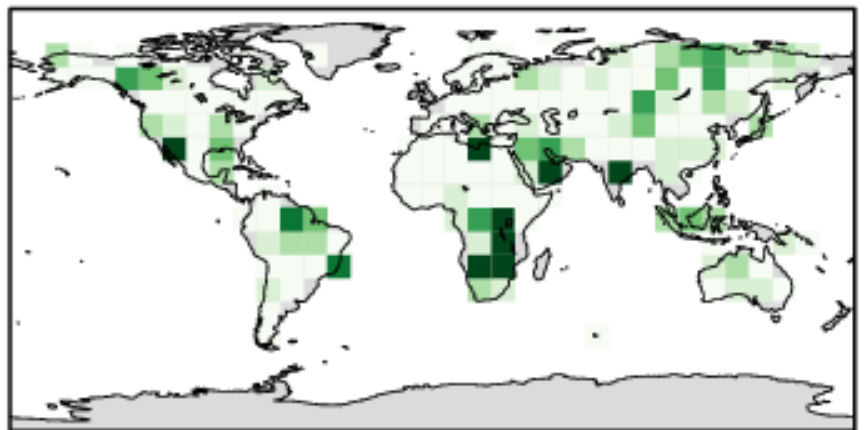

Level 2

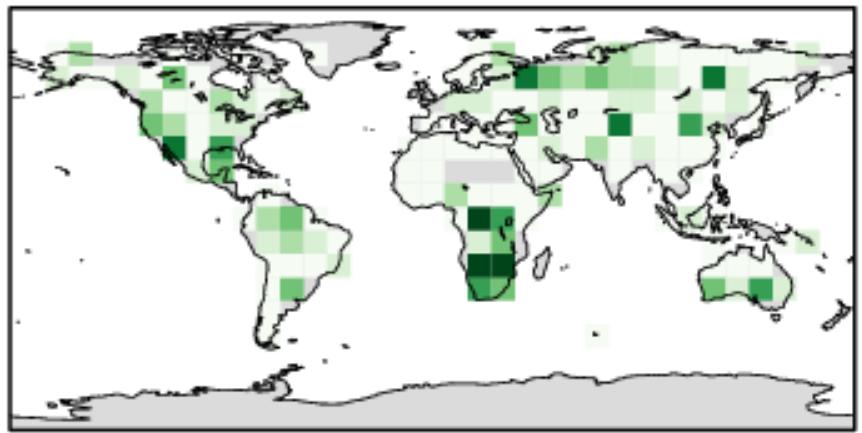

Level 3

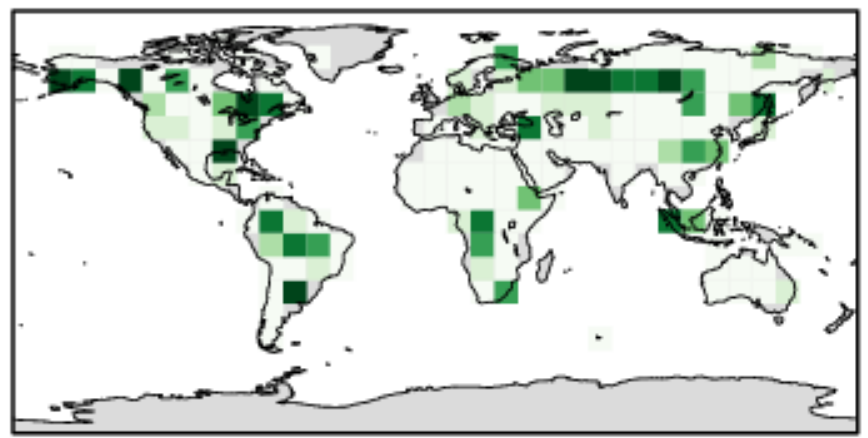

November start

\section{Level 1}

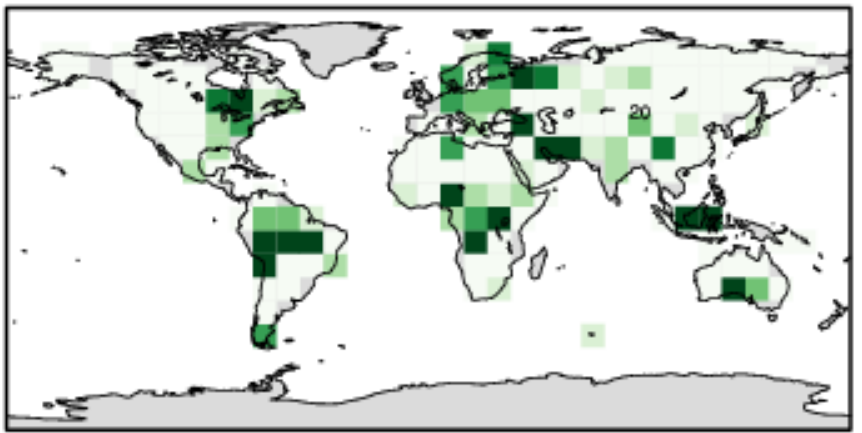

Level 2

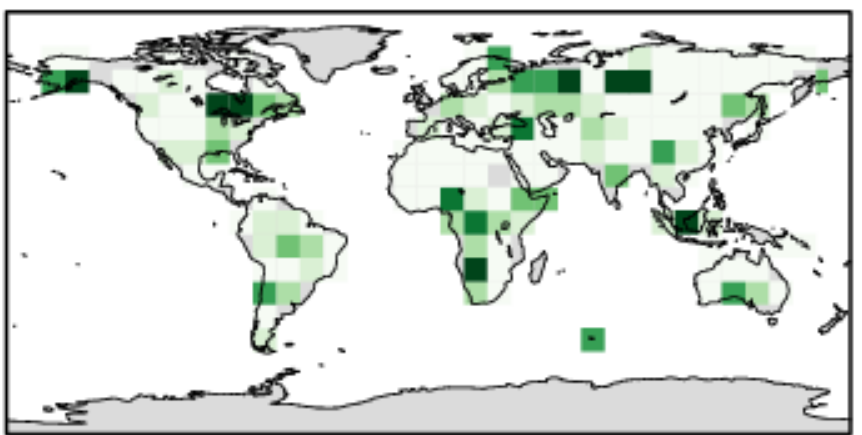

Level 3

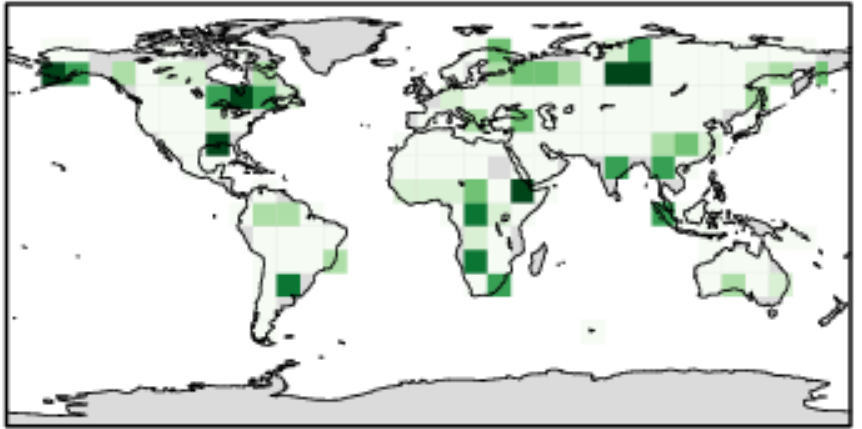

Sensitivity (standard deviation as a percentage of memory loss date) [\%]

\begin{tabular}{rrrrrrr}
\hline & $\mid$ & $\mid$ & $\mid$ & & \\
\hline 20 & 30 & 40 & 50 & 60 & 70
\end{tabular}

Figure 3. Sensitivity of memory to hydraulic parameters (standard deviation of memory loss across hydraulic parameters as a percentage of the memory loss), estimated from HTESSEL, for May (left) and November (right) start dates. 